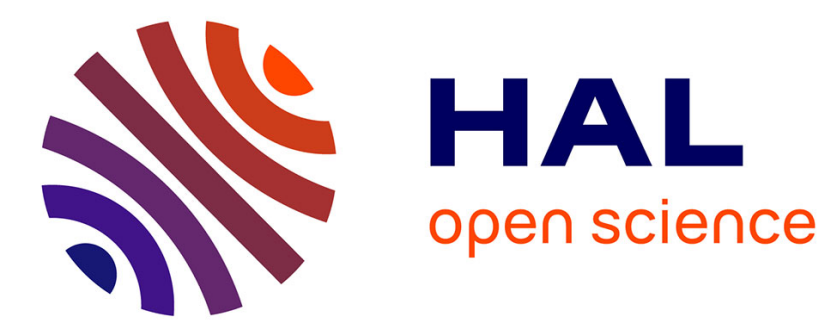

\title{
Plusieurs mots pour une chose. De la nomination multiple au style de Claude Simon
}

Stéphane Bikialo

\section{To cite this version:}

Stéphane Bikialo. Plusieurs mots pour une chose. De la nomination multiple au style de Claude Simon. L'information grammaticale, 2005, 104 (1), pp.50-53. 10.3406/igram.2005.4386 . hal-02306378

\section{HAL Id: hal-02306378 \\ https://hal.science/hal-02306378}

Submitted on 5 Oct 2019

HAL is a multi-disciplinary open access archive for the deposit and dissemination of scientific research documents, whether they are published or not. The documents may come from teaching and research institutions in France or abroad, or from public or private research centers.
L'archive ouverte pluridisciplinaire HAL, est destinée au dépôt et à la diffusion de documents scientifiques de niveau recherche, publiés ou non, émanant des établissements d'enseignement et de recherche français ou étrangers, des laboratoires publics ou privés. 


\section{Plusieurs mots pour une chose. De la nomination multiple au} style de Claude Simon

Stéphane Bikialo

\section{Citer ce document / Cite this document :}

Bikialo Stéphane. Plusieurs mots pour une chose. De la nomination multiple au style de Claude Simon. In: L'Information Grammaticale, N. 104, 2005. pp. 50-53.

doi : 10.3406/igram.2005.4386

http://www.persee.fr/doc/igram_0222-9838_2005_num_104_1_4386

Document généré le 15/12/2015 


\title{
PRÉSENTATION DE THÈSE
}

\author{
PLUSIEURS MOTS POUR UNE CHOSE. \\ DE LA NOMINATION MULTIPLE AU STYLE DE CLAUDE SIMON *
}

\section{Stéphane BIKIALO}

La configuration énonciative de la nomination multiple (NM) consiste en une équivalence (syntaxique et référentielle) entre plusieurs manières de nommer une même chose donnant lieu à des nominations partagées :

et lui, ce résidu de lui-même, ou plutôt cette trace, cette salissure (cet excrément en quelque sorte) laissé derrière soi : dérisoire personnage que l'on voit s'agiter... (1)

Les termes se relativisent mutuellement pour créer une nomination complexe, à la fois dans l'entre-deux et le cumul non hiérarchisé, «ne relevant ni de la coïncidence pleinement confirmée du mot adéquat, ni de la non-coïncidence clairement dénoncée du mot inadéquat » (Authier-Revuz, 1995 : 598). Si la nomination met en relation le réel et la langue par un acte énonciatif, la nomination multiple signale des points de non-coïncidence, d'hétérogénéité montrée. La thèse s'efforce de montrer que ces formes du multiple, qui saturent l'écriture de Claude Simon, représentent un enjeu stylistique, c'est-à-dire à la fois littéraire en ce qui s'y dit quelque chose du rapport du sujet de l'écriture à la langue et au réel, et linguistique en ce que ces formes représentent une configuration énonciative singulière et mettent en scène des «noncoïncidences du dire» (Authier-Revuz). L'approche relève d'une stylistique énonciative au sens où l'on s'attache à une figure énonciative, avant de la considérer dans le style d'une $œ u v r e$. En dépit de son émergence microstructurale, la NM n'est donc pas un procédé mais une manière d'investir la langue. Elle est une figure paradoxale, représentant le manque par l'excès, la difficulté de nommer par la prolifération de nominations. Elle diffère, dédouble la référence, en passant par la voie du multiple contre I'UN. Elle a donc affaire avec le ressassement et le refus du terme (de l'achèvement et du mot juste). Par son caractère de «work in progress » de la nomination (Authier-Revuz, $1995: 616$ ), elle est un mode privilégié d'approche de la textualité simonienne.

À partir d'une conception du style et du sujet de l'écriture, ce travail met en place linguistiquement la configuration de

(*) Thèse de doctorat soutenue le 12 décembre 2003 à l'Université de Poitiers, sous la direction de J. Authier-Revuz (Paris 3 ) et de Ph. Caron (Poitiers), 593 pages. Jury composé de J.-M. Adam (Lausanne), A. Herschberg Pierrot (Paris 8), M. Noailly (Brest) et D. Viart (Lille 3).

1. C. Simon, Le Palace, Minuit, 1962. Nous soulignons. la NM, les formes de langue dans lesquelles elle s'inscrit et ses valeurs énonciatives, avant d'en montrer les enjeux dans l'écriture de C. Simon, en mettant la NM en perspective avec d'autres faits (de niveau macrostructural) et d'autres pratiques d'écriture.

\section{L'EXERCICE DU STYLE}

Partant de l'idée saussurienne selon laquelle «c'est le point de vue qui crée l'objet », cette partie précise le point de vue sur le style qui oriente ce travail. Conçue à la fois comme construction critique et processus, «manière d'être langue » singulière, la notion de style n'est conservée qu'au prix d'une «critique du style », qui ne limite pas le style au microstructural, ni à la « description linguistique du texte littéraire » qui s'arrêterait au seuil de l'interprétation.

Le chapitre 1 propose une articulation du «mode sémiotique », du « mode sémantique » et d'un mode stylistique conçu comme une cristallisation singularisante de l'hétérogénéité discursive au sein d'une œuvre par un sujet. Ce mode stylistique, spécification du mode sémantique, relève de la «translinguistique des textes, des œuvres » (Benveniste, 1974 : 66). La caractérisation de ce mode stylistique s'appuie sur la revendication d'un réel de la langue, à partir duquel peut se penser l'hétérogénéité constitutive de l'énonciation, s'opposant ainsi aux différentes formes de négation de la langue, en stylistique (par l'assimilation langue/norme grammaticale) et dans certaines approches du discours. On insiste en outre sur l'inscription dans des « genres du discours » qui réduisent l'individualité irréductible de tout acte d'énonciation (2). Les « genres du discours » permettent ainsi de penser un champ intermédiaire entre la langue comme système et la phrase comme «événement évanouissant ». C'est le croisement toujours singulier de ces modes d'inscription qui permet de reconnaître un style, pensé comme forme, spécifique, de «sémantisation de la langue » (Benveniste). Cette perspective sémiotique rend caduque toute idée d'écart, le fait de style différant du fait de langue non par nature mais

2. M. Bakhtine (1979), Esthétique de la création verbale, Gallimard, 1984 : «Le locuteur reçoit donc, outre les formes prescriptives de la langue commune [...], les formes non moins prescriptives pour lui de l'énoncé, c'est-à-dire les genres du discours », p. 287. Cf. Adam, 1999. 
par son statut, son inscription dans un contexte discursif (une $œ u v r e$ ). Toutefois, si cette conception sémiotique « est précieuse pour identifier le champ de la singularisation stylistique en général, [elle] ne nous est d'aucun secours pour saisir la singularité d'un style en particulier » (3).

C'est pourquoi le chapitre 2 essaye de cerner la spécificité du mode stylistique à partir d'une réflexion sur la littérarité et le sujet de l'écriture. La subjectivation énonciative laisse place à «l'œuvre en tant qu'espace de singularisation » (Jenny, 1990). Cerner le sujet singulier de l'œuvre c'est s'attacher à son historicité, qui est double (scripteur et lecteur critique). À la suite de Saint-Gérand (1993), on entend par historicité la nécessaire inscription dans une épistémè linguistique et culturelle, une reconstitution critique du sujet de l'écriture. On défend une démarche qui repose sur des positions théoriques tout en s'adaptant à l'historicité de l'œuvre. Le sujet du style est considéré comme un sujet «effet », «sujet «produit» par le langage, comme structurellement clivé par un inconscient, [...] dépossédé de la maîtrise de son dire » (Authier-Revuz, $1995: 66)$. Ce sujet « effet » de la «la langue» lacanienne (registre souterrain de la langue qui la voue à l'équivoque) se distingue à la fois du sujet "vide» de la première analyse de discours (issue de Pêcheux, Foucault et Althusser) et du sujet « origine » de la pragmatique. De cette conception découle un refus du dualisme pensée/langage (4) et une distance très nette prise avec la rhétorique et la stylistique française psychologique ou pragmatique. Est ainsi nuancée la notion non seulement d'écart mais de choix : s'il y a choix, il est en partie inconscient et secondaire, ces « choix imprévus » (Simon) se manifestant dans le cours de l'écriture. Le sujet met en fonctionnement la langue qui le constitue en tant que sujet parlant, dans un mode stylistique d'être langue. Identifier ce rapport du sujet à la langue implique une analyse précise des faits linguistiques et de leur actualisation énonciative.

\section{LA NOMINATION MULTIPLE EN LANGUE ET EN DISCOURS}

Cette partie est consacrée à la description de la nomination multiple comme figure énonciative, expression alternative aux «figures de rhétorique », qui caractérise une configuration linguistique relativement figée, s'appuyant sur des faits de langue spécifiques (aux niveaux morphologique, syntaxique, sémantique), mais nécessitant pour être décrite le recours à l'énonciation. Cette figure est d'abord rencontrée dans l'histoire (chapitre 3), « reconnue » en langue par rapport à d'autres formes et enfin « comprise » en discours, essentiellement chez Simon, dans le double principe de construction du sens qu'elle met en œuvre.

Le chapitre 3 opère un parcours historique des pratiques scripturales et des discours grammaticaux sur des formes s'apparentant à la NM. On évoque ainsi un certain nombre

3. L. Jenny (1993), «L'objet singulier de la stylistique », Littérature $n^{\circ} 89$, p. 120.

4. «On n'écrit jamais quelque chose qui se serait passé (ou pensé) avant que l'on se mette à écrire, mais ce qui se passe (se pense) au présent de l'écriture » (C. Simon). de fonctions discursives qui posent l'horizon interprétatif de la NM ainsi que des prémisses d'analyse syntaxique (Vaugelas et la syntaxe des «synonymes et approchans ») et sémantique (l'abbé Girard et Dumarsais, et les « différences délicates » entre les mots qui préfigurent une analyse saussurienne en terme de différentialité ainsi qu'une analyse du sens en discours). Plusieurs figures de rhétorique prennent en effet en charge une partie des formes de NM, qu'il s'agisse de figures de correction (anthorisme, antéisagoge, épanorthose), de répétition ou d'amplification (métabole, pléonasme, périssologie, synonymie). Les valeurs attribuées à ces figures confrontant plusieurs termes ou syntagmes, sont souvent contradictoires : affaiblissement ou renforcement du premier terme, excès superflu ou énergie du sens. On s'intéresse ainsi à la manière dont la prolifération des doublets synonymiques au MA et au XVIe siècle (clichés de style des récits de voyage) va entraîner leur proscription normative par Malherbe qui dénonce leur caractère ornementaire, redondant, s'opposant au mot juste, proscription qui perdure chez Marouzeau et Cressot, au sujet d'auteurs comme Huysmans, Proust, Péguy... La réflexion sur la synonymie qui prend son essor au XVIIIe siècle, avec la distinction entre synonymie accumulative (liée à l'esthétique de l'emphase) et distinctive (liée à l'esthétique du mot juste), permet de préciser le double horizon normatif de la NM entre mot en trop et recherche du mot juste.

Le chapitre 4 définit la relation d'équivalence syntaxique, sémantique et référentielle à l'œuvre dans la NM. Cette figure énonciative relève d'une prédication d'équivalence instituée en discours et immédiate sur la chaîne, à la différence de la réécriture. Outre une réflexion sur la coordination, on s'attache particulièrement aux structures juxtaposées, cadre ambigu (Soublin, Tamine, 1973) quant à l'équivalence syntaxique. Le statut de figure énonciative de la NM permet d'établir moins des critères que des indices d'équivalence. On peut distinguer la NM des constructions détachées (5) et en particulier de l'apposition, «relation de dépendance syntacticosémantique unilatérale »(Neveu, 1998 : 67). Le détachement et la prédication seconde instaurent une dissymétrie syntaxique qui les distingue de la relation en jeu dans la NM, reposant sur une coréférence forte liée à l'équivalence (6). Les cadres syntaxiques s'avérant non univoques, on s'appuie sur des indices tels que l'instanciation d'une même place syntaxique, l'accord et surtout les déterminants. Dans la NM, les déterminants sont symétriques, disposent d'une charge référentielle équivalente tout en ayant un référent unique (7). L'ordre des mots ne paraît pas pertinent dans la mesure où «le parallélisme institué par la syntaxe tend à se projeter sur la relation sémantique et à neutraliser la

5. Par rapport aux CD, «expressions non référentielles », traitées par B. Combettes (1998), Les Constructions détachées en français (Ophrys), les NM sont des expressions référentielles.

6. Selon M. Forsgren (1991) toutefois, la NM représente le "prototype » de l'apposition («Éléments pour une typologie de l'apposition en linguistique française ", Actes du XVIIle Congrès International de Linguistique et de Philologie Romanes, t. 2, Max Niemeyer).

7. Ce qui situe la NM à la jonction de la juxtaposition énumérative, où il y a deux actualisations car deux référents distincts, et de l'apposition avec une actualisation et une anaphore (Tamba-Mecz, 1975 : 248). 
hiérarchie des deux segments " (Noailly, 2000 : 59). L'équivalence sémantico-référentielle, elle, peut être décrite à partir de la « référence virtuelle » de Milner, qui renvoie au « type des segments de réalité possibles qui peuvent ou non, moyennant un acte d'énonciation, être désignés " par une unité lexicale (8), et permet donc d'articuler les deux ordres de la langue et du réel. La référence virtuelle renvoie à l'ambivalence de tout mot à la fois tourné vers le monde et vers les autres signes dans la langue. Pour préciser la nature de cette articulation, on distingue le « réel » et la « réalité », qui fait d'une chose un « référent » à travers un « acte de dénomination» (Kleiber, 1984). La nomination, acte énonciatif occurrent, s'appuie sur cette dénomination instituée.

L'équivalence syntaxique et référentielle permet de distinguer les figures du multiple (apposition, comparaison, coordination énumérative) des divers types de NM :

a) la NM par juxtaposition (cette trace, cette salissure), où les deux manières de dire créent, en l'absence de marqueur, une sorte de syntagme complexe, pouvant aller jusqu'à la création d'un "substantif épithète " (homme-cheval);

b) la NM par reformulation paraphrastique, marquée par c'est-à-dire (le capitaine, c'est-à-dire le géant) (9), ou plutôt, non pas... mais, ou le ou d'équivalence (quelque poussée, quelque convulsion ou dilatation) (10);

c) la NM avec comme métalinguistique d'approximation, où le comme est supprimable et remplaçable par pour ainsi dire (un illusoire et ultime intervalle de vide, comme un fossé, un étroit canyon ou plutôt une invisible muraille);

d) la NM parenthétique, cas particulier de décrochement, qui «annule le critère de suppressibilité » de la parenthèse (Pétillon, 2003 : 166), dans la mesure où l'élément $X$ est soit gardé comme appui, soit plus fort référentiellement (ils (les cavaliers) étaient en train de subir).

Ces formes du multiple se distinguent au niveau du mode de référenciation : elles présentent des références hiérarchisées, les NM parenthétiques, reformulatives et avec comme des "références contiguës ", et les NM par juxtaposition des « références homogènes » (Noailly, $1990: 81$ ).

Dans le chapitre 5, sont décrits deux mouvements du sens principaux : la construction dynamique du sens et la construction du sens dans un entre-deux. Intervenant dans tout discours, ils permettent néanmoins d'envisager certaines spécificités du style de Simon. Par le premier mouvement, est mis en avant l'exhibition dans l'écriture de la construction de la référence, la présence du sujet à travers les retours méta-énonciatifs que constitue la NM, ainsi que le jeu des mots, l'avancée dans la langue, avec le passage d'un mot à un autre fondé sur le signifiant :

ce hautain orgueil de classe ou plutôt de caste (L'Acacia, Minuit, $1989: 127)$

8. J.-C. Milner (1978), De la syntaxe à l'interprétation. Quantités, insultes, exclamations, Seuil, p. 26.

9. Est exclu de la NM le c'est-à-dire reliant deux faits, qui relève de la « reprise interpérative » (Murat, Cartier-Bresson, 1987, “C'EST-À-DIRE ou la reprise interprétative ", Langue française $\mathrm{n}^{\circ} 73$ ).

10. Voir I. Tamba (1987), " "Ou" dans les tours du type : "un bienfaiteur public ou évergète" ", Langue française $n^{\circ} 73$.
Ce mode de construction du sens induit une rareté des occurrences de NM marquant un mouvement de spécification, une rectification, les cas de spécification étant souvent un leurre, la NM introduisant des métaphores, des expansions rendant la référence problématique au lieu de la spécifier. Le second mouvement (l'intervalle du sens), où la nomination relève de l'entre-deux, fait jouer le Même dans l'Autre (énumérations à référents distincts rapprochés) et l'Autre dans le Même (par la NM) donnant lieu des tensions thématiques et scripturales propres à Simon (immobilité/mouvement, brouillages temporels). C'est le mode de progression du texte qui est ainsi métaphorisé. La NM inscrit le texte sous le régime de l'approximation, où la référence est toujours suspendue entre deux mots ce qu'illustrent les comme et les enclosures, qui situent la nomination dans un dire « faute de mieux ". Cette approximation peut passer par des modalités suspensives du dire (cette - mais comment dire? : battue, poursuite, traque, farce, hallali ?) et où il avait joué le rôle de gibier). C'est enfin entre deux homonymes ou termes métaphoriques que peut se situer la nomination., reposant sur l'intervalle entre les signes.

Deux chemins possibles semblent pouvoir être distingués à partir des formes de NM : le chemin de l'aiguilleur (d'un mot à un autre) et celui du poinçonneur (poinçonnages successifs du réel par des nominations insatisfaisantes). Leur superposition ou les glissements de l'un à l'autre sont l'une des spécificités du style de Simon.

\section{ENTRE LES MOTS ET LES CHOSES POUR AINSI DIRE : SUR LE STYLE DE CLAUDE SIMON}

Tout en convoquant l'ensemble du corpus simonien, et en le mettant en perspective avec d'autres pratiques d'écriture (N. Sarraute, F. Ponge, R. Antelme), les analyses sont concentrées sur La Route des Flandres, Le Palace et L'Acacia. La partie est fondée sur l'idée d'équilibre entre la dimension littérale et référentielle. Le style de Simon est abordé du point de vue privilégié de la NM considérée comme structure révélatrice d'un rapport du sujet à la langue et au réel.

Dans le chapitre 6 sont essentiellement abordés les rapports entretenus par cette écriture avec la linéarité et son refus de la nomination UNE. On insiste sur le fait que son style ne s'oppose pas à la langue mais aux normes, et que son «illisibilité " souvent évoquée vient de ce que la norme (moyenne des usages) s'oppose à la mise en jeu des virtualités de la langue. Le rapport à la linéarité est emblématique de ce rapport à la langue : l'écriture simonienne rompt rarement avec la linéarité mais exploite les différents types de compromis avec elle, cherchant à y inscrire ce qui relève de l'architecture sensorielle, par la projection du paradigme sur le syntagme et le rythme de la simultanéité conséquent à l'absence de hiérarchisation de la NM. Ajout linguistique, la NM se présente comme une rature représentée proche de l'ajout génétique (Herschberg Pierrot, 2002), rapprochant Simon de Ponge et de son «nouveau genre littéraire», le «moviment ». Ces valeurs de la NM, mises en relation avec la diégèse et la structure de L'Acacia, permettent de montrer 
comment l'œuvre creuse un même paradigme (virtuel), associatif (mémoriel), mettant en jeu une mémoire imparfaite qui relance l'écriture et que l'écriture relance sur le mode de la quête inachevée. En plus de ces rapports multiples à la linéarité, la NM met en scène un style de l'entre-deux qui se retrouve dans Le Palace par le refus de la nomination UNE et dans La Route des Flandres par le statut de Georges à la croisée des discours. L'inquiétude de la langue se manifeste en effet par la manière dont la NM la fait jouer comme lieu d'équivoque. Dans Le Palace, la NM souligne le leurre de la recherche du UN de la nomination et du Même entre deux époques chez le personnage, mais dénonce aussi le leurre contraire de l'Autre chez les révolutionnaires; c'est dans l'entre-deux que le roman se situe, au niveau des personnages, de la structure du roman, et des époques. Dans $\mathrm{La}$ Route des Flandres, l'entre-deux n'est plus temporel mais interdiscursif : le c'est-à-dire omniprésent semble renvoyer à une norme d'expression qui dissimule un investissement du texte par lalangue et l'interdiscours. Cette dimension d'excès de la langue confère à cette œuvre son caractère d'énigme, cette forme simple jouant précisément des «mots sous les mots ». L'écriture simonienne exemplifie ainsi le fait que «la langue reprend tout dans ses possibilités » (Deguy), l'expérience et le caractère informe du réel n'étant pas indicibles mais nécessitant un travail dans la langue, une tentative d'épuisement des possibles langagiers.

Le chapitre 7 aborde la question du rapport au réel pour montrer que le refus du réalisme chez Simon ne relève en rien d'un refus du réel, et qu'au contraire la représentation dans l'écriture d'une «non-coïncidence entre les mots et les choses » (Authier-Revuz) est une manière de se rapprocher du réel, dans une écriture non pas « réaliste » mais « réeliste » (11). L'écriture de Simon revendique une hétérogénéité entre les mots et les choses, et la thématise par la mise en œuvre de filtres entre le réel et les mots (perceptions, mémoire, représentations mythiques, picturales, photographiques et littéraires), le filtre le plus récurrent étant celui de la «pellicule de poussière », métaphore filée qui parcourt l'œuvre. Être au plus près du réel consiste donc à le dire dans sa non-coïncidence aux mots, et à souligner la nécessité de passer par la réalité, les représentations culturelles et les nominations instituées qui conditionnent toute approche du réel (cf. Viart, 1997) :

dix jours de combat, ou plutôt de retraite, ou plutôt de chasse à courre où il - le jeune homme de bonne famille - avait [...] tenu le rôle de gibier (La Route des Flandres, Minuit, 1960 : 159)

et non pas en pleine retraite ou plutôt débâcle ou plutôt désastre (ibid. : 16)

Dans ces NM en écho, la nomination est partagée au sens où elle est située dans l'intervalle entre les trois mots, ce que confirme une étude des relations sémantiques : retraite possède ainsi un sens en vénerie, qui permet de glisser vers chasse à courre. Dans le second extrait, plus que l'effet de surenchère c'est la réponse de la langue (par la proximité des signifiants) qui apparaît, faisant passer de la non-coïncidence des mots et des choses à celles des mots à euxmêmes. La NM peut ainsi apparaître comme une forme de tentative « d'épuisement » du réel (Rabaté) qu'on nomme «réelisme». Tenter d'épuiser le réel, c'est moins chercher à s'en débarrasser en l'écrivant que le faire exister, le préserver en pointant des détails qui en rompent le caractère amorphe et discontinu. La tentative d'épuisement ne peut mener qu'au ressassement car cette tentative se dit forcément dans le manque du langage, toujours relancé vers d'autres dires, problématique qui rapproche Simon de la littérature dite des camps.

Manifestation ponctuelle de cet « autre versant du langage » (Aquien, 1997) que représente le style, la nomination multiple met diversement en œuvre chez Simon cette formule de Ponge : « II faut beaucoup de mots pour détruire un seul mot (ou plutôt pour faire ce mot non plus un concept mais un conceptable) ».

\section{Stéphane BIKIALO Université d'Amiens Forell}

\section{RÉFÉRENCES BIBLIOGRAPHIQUES}

Adam J.-M. (1999), Linguistique textuelle: des genres de discours aux textes, Nathan.

Aquien M. (1997), L'Autre versant du langage, Corti.

Authier-Revuz J. (1987), « L'auto-représentation opacifiante du dire dans certaines formes de "couplage" ", DRLAV n³6-37, p. 55-103.

Authier-Revuz J. (1995), Ces mots qui ne vont pas de soi. Boucles réflexives et non-coïncidences du dire, tomes 1 et 2, Larousse.

Benveniste E. (1966) (1974), Problèmes de linguistique générale, t. 1 et 2, Gallimard, « Tel».

Boucheron-Petillon S. (2003), Les Détours de la langue. Étude sur la parenthèse et le tiret double, Peeters, «BIG».

Herschberg Pierrot A. (2002), «Ajout génétique et ajout linguistique », Figures d'ajout (dir. J. Authier-Revuz et M.-C. Lala), Presses de la Sorbonne Nouvelle, p. 34-39.

Jenny L. (1990), La Parole singulière, Belin.

Kleiber G. (1984), «Dénomination et relations dénominatives », Langages $n^{\circ} 76$, p. 77-94.

Neveu F. (1998), Études sur l'apposition. Aspects du détachement nominal et adjectival en français contemporain dans un corpus de textes de J.-P. Sartre, Champion.

Noailly M. (1990), Le Substantif épithète, PUF, « Linguistique nouvelle».

Noailly M. (2000), «Apposition, coordination, reformulation dans les suites de deux GN juxtaposés », Langue française $\mathrm{n}^{\circ} 125$, p. 46-59.

Rabate D. (1991), Pour une littérature de l'épuisement, Corti.

Saint-Gérand J.-Ph. (1993), Morales du style, P.U. Mirail.

Saussure F. de (1916), Cours de linguistique générale, Payot, 1972.

Soublin F., Tamine J. (1973), « Métaphores et cadres syntaxiques : la juxtaposition », Le Français moderne XLI, n 3, p. 240-255.

Tamba-Mecz I. (1975), «Système de l'identification métaphorique dans la construction appositive », Le Français moderne XLIII, $\mathrm{n}^{\circ} 3$, p. 234-255.

Viart D. (1997), Une mémoire inquiète. La Route des Flandres de Claude Simon, PUF, «Écrivains ».

11. Cf. J.-M. Gleize (2000), «Prenez et mangez (fragments d'un manifeste réeliste) ", La Licorne ${ }^{\circ} 53$. L'opposition réalisme/réelisme prend appui sur celle posée entre réel et réalité (chap. 4). 